\title{
Quantum teleportation and entanglement distribution over 100-kilometre free-space channels
}

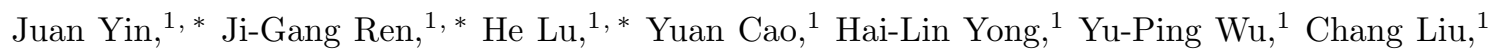 \\ Sheng-Kai Liao, ${ }^{1}$ Fei Zhou, ${ }^{1}$ Yan Jiang, ${ }^{1}$ Xin-Dong Cai, ${ }^{1}$ Ping Xu,${ }^{1}$ Ge-Sheng Pan, ${ }^{1}$ Jian-Jun Jia, ${ }^{2}$ \\ Yong-Mei Huang, ${ }^{3}$ Hao Yin, ${ }^{1}$ Jian-Yu Wang, ${ }^{2}$ Yu-Ao Chen, ${ }^{1}$ Cheng-Zhi Peng, ${ }^{1}$ and Jian-Wei Pan ${ }^{1}$ \\ ${ }^{1}$ Shanghai Branch, National Laboratory for Physical Sciences at Microscale and Department of Modern Physics, \\ University of Science and Technology of China, Shanghai 201315, China \\ ${ }^{2}$ Shanghai Institute of Technical Physics, Chinese Academy of Sciences, Shanghai 200083 China \\ ${ }^{3}$ The Institute of Optics and Electronics, Chinese Academy of Sciences, Chengdu 610209, China
}

(Dated: January 29, 2013)

\begin{abstract}
A long standing goal for quantum communication is to transfer a quantum state over arbitrary distances. Free-space quantum communication provides a promising solution towards this challenging goal. Here, through a $97-\mathrm{km}$ free space channel, we demonstrate long distance quantum teleportation over a 35-53 dB loss one-link channel, and entanglement distribution over a 66-85 dB high-loss two-link channel. We achieve an average fidelity of $80.4(9) \%$ for teleporting six distinct initial states and observe the violation of the Clauser-Horne-Shimony-Holt inequality after distributing entanglement. Besides being of fundamental interest, our result represents a significant step towards a global quantum network. Moreover, the high-frequency and high-accuracy acquiring, pointing and tracking technique developed in our experiment provides an essential tool for future satellite-based quantum communication.
\end{abstract}

PACS numbers:

The concept of quantum entanglement, a fundamental feature of quantum mechanics, lies at the heart of quantum information processing [1. With the help of quantum entanglement among distant locations, quantum communication (QC) can be achieved over arbitrary distances by quantum teleportation and entanglement distribution. The discovery of the former 2 has led to seminal quantum information processing protocols like large-scale $\mathrm{QC}[3]$, and the latter has become an indispensable process in many $\mathrm{QC}$ schemes such as entanglement based quantum key distribution [4] and test of non-locality of quantum mechanics $[5]$.

Free-space optical quantum channel appears to be very promising to implement long-distance QC. As information carrier photons are fast and robust. Since the photon loss is almost negligible in the outer space and the effective thickness of the atmosphere is only about $10 \mathrm{~km}$, free-space optical channel, first used for quantum key distribution [9, 10, can be far superior over fiber links and is most promising for satellite-based QC on a global scale.

Quantum teleportation was first demonstrated with entangled photons[11] in 1997. Later, various developments have been achieved in laboratory, including the demonstration of entanglement swapping[12], opendestination teleportation[13] and teleportation of twobit composite system [14. Entanglement distribution has been shown with fiber links 15-18. In addition, "practical" quantum teleportation have been realized via fiber links [19, 20] and limited to a distance of about one kilo-

\footnotetext{
${ }^{*}$ These authors contributed equally to this work
}

meter. Experiments have achieved free space distribution of entangled photon pairs over distances of $600 \mathrm{~m}[21$ and $13 \mathrm{~km} 22$. Later, entangled photons were transmitted over $144 \mathrm{~km} 23,24]$. In these experiments, either only one photon was transmitted [23] or the entangled photon pair was transferred together 24 using only a one-link channel. Most recently, following a modified scheme 25], quantum teleportation over a $16 \mathrm{~km}$ free-space links was demonstrated [26] with a single pair of entangled photons. However in this experiment, the unknown quantum state must be prepared on one of the resource entangled qubits and therefore cannot be presented independently.

In our experiment, we first demonstrate quantum teleportation of an independent unknown state through a 97$\mathrm{km}$ one-link optical free-space channel with multi-photon entanglement, following the original scheme 2, 11. An average fidelity of $80.4(9) \%$ can be achieved for six distinct initial states over a 35-53 dB loss quantum channel. Furthermore we report an entanglement distribution over a two-link quantum channel, where the entangled photon pairs are separated by $101.8 \mathrm{~km}$ and the total channel loss is up to 66-85 dB. Violation of the Clauser-HorneShimony-Holt (CHSH) inequality 27] is successfully observed. The successful quantum teleportation and the entanglement distribution over such channel losses in combination with our high-frequency and high-accuracy acquiring, pointing and tracking (APT) technique show the feasibility of satellite-based ultra-long-distance quantum teleportation and global-scale QC. 


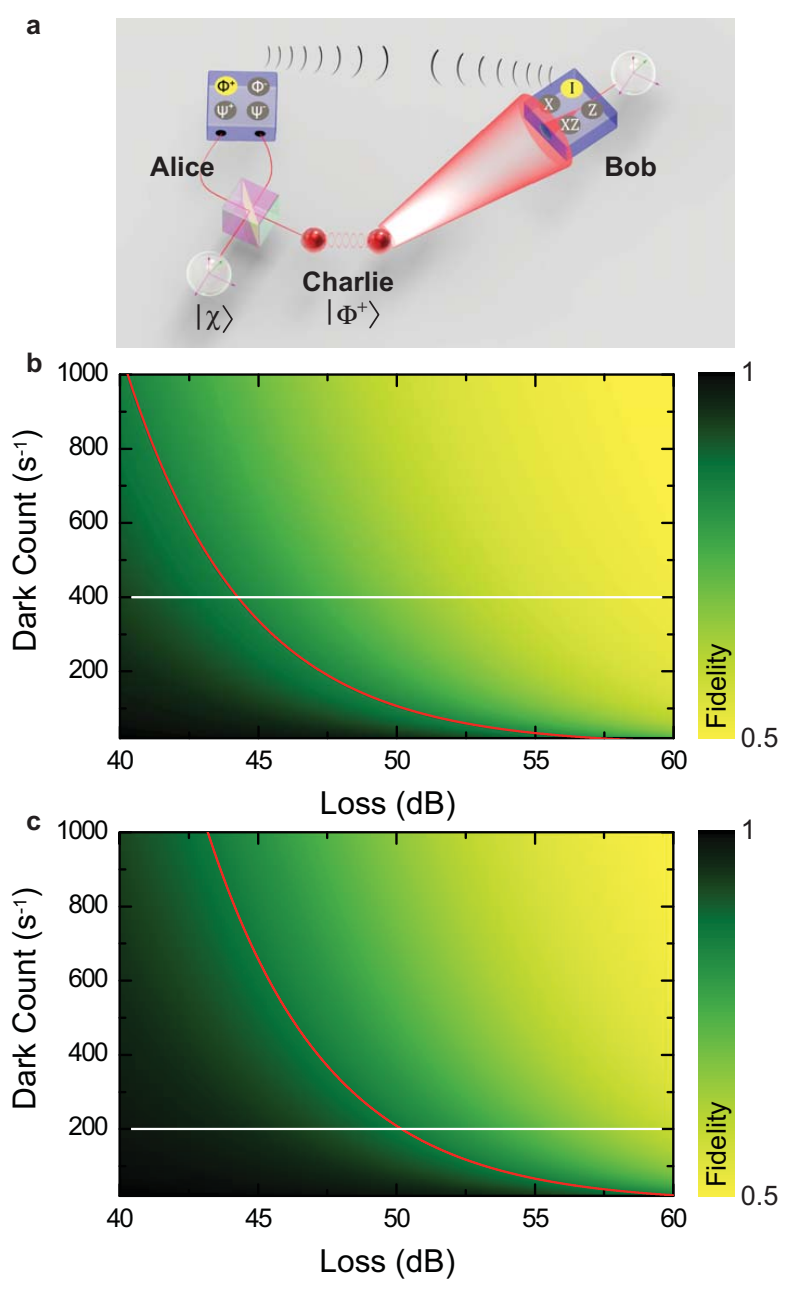

FIG. 1: Experimental scheme and calculated fidelity for practical free-space quantum teleportation. a Schematic drawing for practical free-space quantum teleportation. Charlie distributes an entangled pair of photons 2 and 3 to Alice and Bob, where Bob is at a distant location. Due to the finite size of the telescopes Bob and Charlie can use and the diffraction limit, Bob will receive the signal photon with very high loss. Alice then performs a joint Bell-state measurement (BSM) on the initial particle and one of the entangled photons from Charlie, projecting them onto an entangled state. After she has sent the result of her measurement as classical information to Bob, he can perform a unitary transformation (U) on his photon to obtain the initial state. Note that in our experiment, the unitary transformation was not applied. $\mathbf{b}$ Calculated fidelity for ideal optics and an entangled photon source with the generation probability of 0.083 pair per pulse and overall detection efficiency of $12.3 \%$ used in Ref. 28] as a function of total channel loss and the dark count on Bob's side. The red line shows the classical limit 29] of $2 / 3$. The white line shows the lowest dark count rate currently achieved via free-space link 24]. c Calculated fidelity for ideal optics for our ultra-bright entangled photon source with the generation probability of 0.1 pair per pulse and overall detection efficiency of $23.6 \%$.

\section{Teleportation through one-link channel}

A schematic illustration of free-space quantum teleportation is shown in Fig. 17. Alice has a photon in an unknown quantum state $|\chi\rangle_{1}=\alpha|H\rangle+\beta|V\rangle$, where $H(V)$ represents the horizontal (vertical) polarization. Alice wants to transfer the photon to Bob, who is at a distant location. To do so, Charlie first distributes an entangled photon pair 2 and $3,\left|\Phi^{+}\right\rangle_{23}=(|H\rangle|H\rangle+|V\rangle|V\rangle) / \sqrt{2}$, to Alice and Bob, respectively. The combinative state of the three photons can be rewritten as

$$
\begin{gathered}
|\chi\rangle_{1} \otimes\left|\Phi^{+}\right\rangle_{23}=\frac{1}{2}\left(\left|\Phi^{+}\right\rangle_{12}|\chi\rangle_{3}+\left|\Phi^{-}\right\rangle_{12}\left(Z|\chi\rangle_{3}\right)+\right. \\
\left.\left|\Psi^{+}\right\rangle_{12}\left(X|\chi\rangle_{3}\right)+\left|\Psi^{-}\right\rangle_{12}\left(X Z|\chi\rangle_{3}\right)\right)
\end{gathered}
$$

where $\left|\Phi^{ \pm}\right\rangle_{12}=\left(|H\rangle_{1}|H\rangle_{2} \pm|V\rangle_{1}|V\rangle_{2}\right) / \sqrt{2},\left|\Psi^{ \pm}\right\rangle_{12}=$ $\left(|H\rangle_{1}|V\rangle_{2} \pm|V\rangle_{1}|H\rangle_{2}\right) / \sqrt{2}$ are the four Bell states, and $\mathrm{Z}, \mathrm{X}$ are Pauli operators, which act as unitary transformations. Then, if Alice performs a joint Bell-state measurement (BSM) on her two photons, photon 3 is instantaneously projected into the four states $\left|\chi_{3}\right\rangle, Z\left|\chi_{3}\right\rangle$, $X\left|\chi_{3}\right\rangle$ and $X Z\left|\chi_{3}\right\rangle$, respectively. Thus, after receiving the BSM result from Alice via a classical channel, Bob can apply the appropriate unitary transformation to convert the state of photon 3 to the initial state 2, 11.

Practically, the quantum channel between Charlie and Bob always has losses due to the finite size of the telescopes on both sides (see Fig. 11). Typically, for an uplink of the ground station to a satellite, this loss can be up to $45 \mathrm{~dB}$ (calculated for $20 \mathrm{~cm}$ satellite optics at an orbit height of $500 \mathrm{~km}$ ). The losses themselves only reduce the success probability of the teleportation. With perfect detectors and without background light, the channel losses would not be the limiting factor. However, because of the intrinsic dark counts by the detector and additional background from the environment, there is a chance that a dark count produces an error each time a photon is lost. When the probability of a dark count becomes comparable to the probability that a photon is correctly detected, the signal-to-noise ratio tends to 0 . In order to overcome this problem, one has two possibilities: increasing the brightness of the entangled photon source or reducing the dark count rate. The calculated fidelity as a function of dark count rate and channel loss is shown in Fig. 1p. One can see that even with ideal optics, it is not possible to demonstrate successful quantum teleportation under a $45 \mathrm{~dB}$ loss channel with the currently achieved lowest dark count rate 24] of $\sim 400 \mathrm{~s}^{-1}$.

Experimentally, we start with an ultra-bright entangled photon source 30 based on type-II spontaneous parametric down-conversion 31] (SPDC). As shown in Fig. 2 a, on Charlie's side (located at Gangcha next to Qinghai Lake, $37^{\circ} 16^{\prime} 42.41^{\prime \prime}$ N $99^{\circ} 52^{\prime} 59.88^{\prime \prime} \mathrm{E}$, altitude $3262 \mathrm{~m}$ ), a femtosecond ultraviolet (UV) laser is created by frequency doubling a pulsed laser (MIRA, central wavelength of $788 \mathrm{~nm}$ with a duration of $130 \mathrm{fs}$ and a repetition rate of $76 \mathrm{MHz}$ ) with an $\mathrm{LBO}$ crystal 


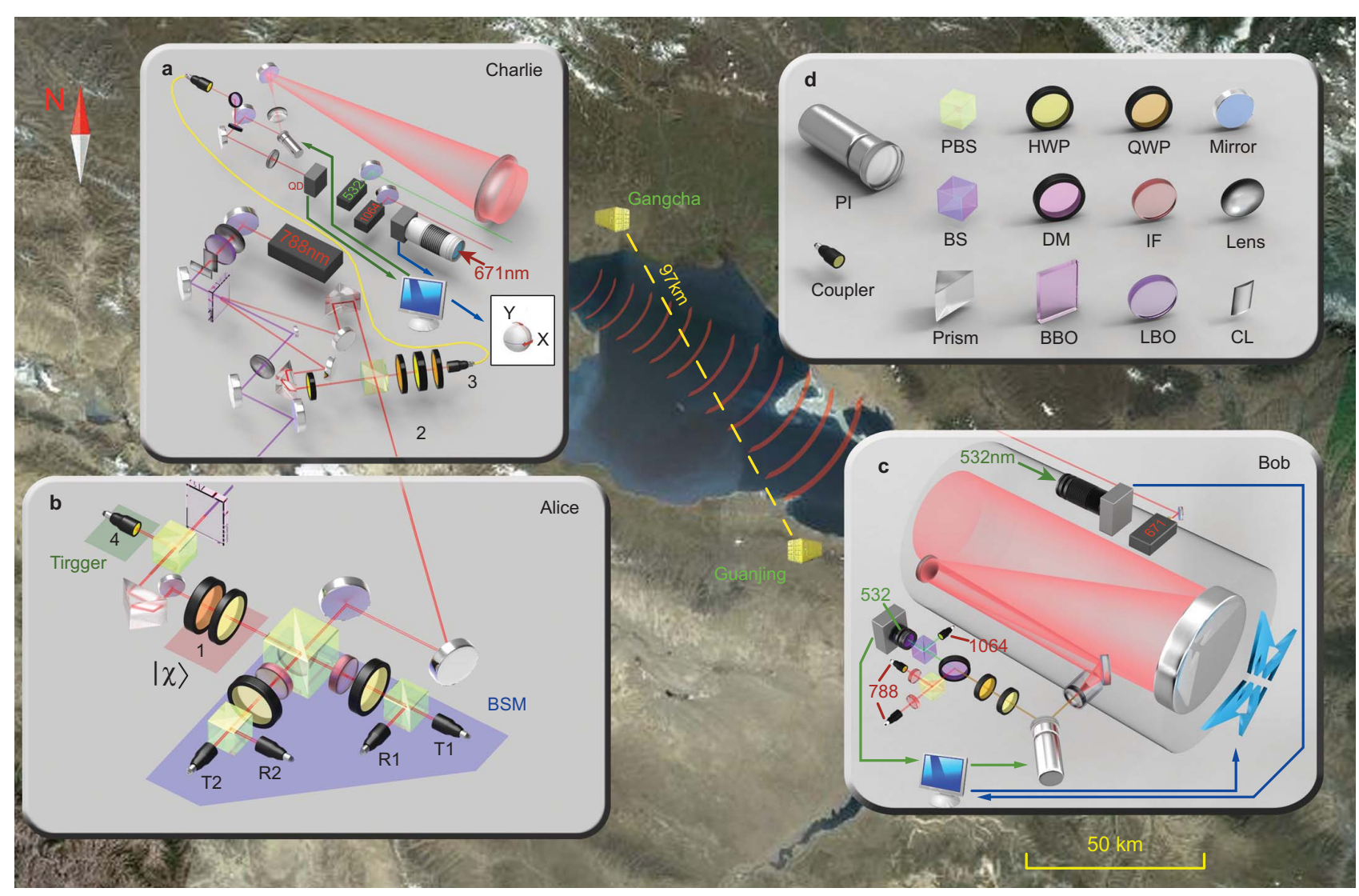

FIG. 2: Bird's-eye view and schematic diagram for free-space quantum teleportation. a, Entanglement generation and distribution on Charlie's side. A near infrared pulse $(788 \mathrm{~nm})$ is focused on an LBO crystal to create an ultraviolet laser pulse, which is then focused with two cylindrical lenses (CL) and passed through a $2 \mathrm{~mm}$ nonlinear BBO crystal. By an SPDC process, an entangled photon pair is created. An interferometric Bell-state synthesizer is utilized to disentangle the temporal from the polarization information 30 . While photon 2 is then directly sent to Alice for a BSM, photon 3 is guided to a refractor telescope through a fiber and sent to Bob. An HWP sandwiched between two QWPs constitute the fiber polarization compensation. Coaxial with the telescope, there is a green laser (532 nm) for system tracking and a infrared laser (1064 nm) for synchronization. The green arrows indicate the fine tracking system which consists of a four-quadrant detector (QD) and a fast steering mirror driven by piezo ceramics (PI). The blue arrows indicate the coarse tracking system which consists of a wide-angle camera and a two-dimensional rotatable platform. b, Initial state preparation and BSM on Alice's side. Alice sends the UV laser through a collinear BBO, creating another photon pair which is later separated in path by a PBS. An HWP and a QWP is applied in path 1 to prepare the initial unknown quantum state to be teleported. Alice interferes the initial state 1 and the photon 2 from Charlie using another PBS. A $22.5^{\circ} \mathrm{HWP}$ and PBS is placed at both outputs for polarization analysis. A coincidence between detectors T1 and T2 (R2) or R1 and R2 (T2) indicates the incident state of $\left|\Phi^{+}\right\rangle\left(\left|\Phi^{-}\right\rangle\right)$. c, Polarization analysis on Bob's side. Bob receives photon 3 with a $400 \mathrm{~mm}$ diameter off-axis reflecting telescope. A polarization analyzer is assembled at the telescope's exit, containing an HWP, a QWP, a PBS, and two multi-mode fiber-coupled SPCMs. Coaxial with the receiving telescope, there is another high-power beacon laser $(671 \mathrm{~nm})$ for system tracking. The blue and green arrows indicate the coarse and fine tracking system, respectively. d, Symbols used for the setup.

$\left(\mathrm{LiB}_{3} \mathrm{O}_{5}\right)$. The UV laser is further guided to pump a noncollinear type-II $\beta$-barium (BBO) crystal, resulting in a pair of polarization entangled photons in the state $|\Psi\rangle=\left(\left|H_{o} V_{e}\right\rangle+\left|V_{e} H_{o}\right\rangle\right) / \sqrt{2}$ with temporal and polarization information also entangled [30, where $o$ and $e$ indicate the polarization with respect to the pump. With an interferometric Bell-state synthesizer 30, 32, we disentangle the temporal from the polarization information by guiding photons of different bandwidths through separate paths, resulting in the desired entangled photon source $\left|\Phi^{+}\right\rangle_{23}=(|H H\rangle+|V V\rangle)_{23} / \sqrt{2}$. Charlie then distributes the two photons 2 and 3 to Alice and Bob, respectively.

To prepare the unknown quantum state to be teleported, Alice uses the UV laser to pump a collinear BBO crystal which emits photons along the pumping light direction (see Fig. $2 \mathrm{~b}$ ). The generated photons are $|H V\rangle_{14}$, which are then split by a PBS after the pumping laser has been filtered out. A half wave plate (HWP) and a quarter wave plate (QWP) are applied in path 1 to create the initial state. Under a trigger on path 4, Alice creates the state she wants to teleport. Then, Alice performs 
a joint BSM on photon 1 and 2 by interfering them on a PBS and performing polarization analysis on the two outputs. The subsequent coincidence measurements can identify the $\left|\Phi^{ \pm}\right\rangle$Bell states in our experiment.

In the experiment, the power of the pulsed UV laser was about $1.3 \mathrm{~W}$ after frequency doubling. The observed average two-fold coincidence rate was $4.4 \times 10^{5} \mathrm{~s}^{-1}$ with $3 \mathrm{~nm}$ filters in the $e$-ray path 2 and $8 \mathrm{~nm}$ filters in the $o$-ray path 3 . The visibility of the entangled photon pair was about $91 \%$ in the $|H\rangle /|V\rangle$ basis and $90 \%$ in the $|+\rangle /|-\rangle$ basis, where $| \pm\rangle=1 / \sqrt{2}(|H\rangle \pm|V\rangle$. The generation rate of the entangled photons was about 0.1 pair per pulse, and the overall detection efficiency was 0.236 locally. The count rate of photon pair $|H V\rangle_{14}$ generated by the collinear BBO crystal was $6.5 \times 10^{5} \mathrm{~s}^{-1}$, with the photon in path 1 (e-ray) filtered by an interference filter $\left(\Delta_{\mathrm{FWHM}}=3 \mathrm{~nm}\right)$ and the photon in path 4 (o-ray) detected without filters. In the joint BSM, the observed visibility of interference for the photons overlapping on the PBS was 0.6. Finally, we observed about a $2 \times 10^{3} \mathrm{~s}^{-1}$ counting rate for four-fold coincidence locally. As comparison, we plot the calculated fidelity as a function of dark count rate and channel loss for our source (Fig. 1.

On the other hand, Charlie sends photon 3 with a compact transmitting system to Bob on the other side of Qinghai Lake, as shown in Fig. 2 a. A $127 \mathrm{~mm} \mathrm{f/7.5}$ extra-low dispersion alternative public offering refractor telescope is employed as an optical transmitting antenna. By minimizing the color dispersion, we obtain superior sharpness and color correction. For near-diffractionlimited far-field divergence angles, we design systems to substantially reduce chromatic and spherical aberrations. Finally, the divergence angle of our compact quantum transmitter is about $20 \mu \mathrm{rad}$.

As shown in Fig. 2e, Bob (GuanJing $36^{\circ} 32^{\prime} 43.31^{\prime \prime} \mathrm{N}$ $100^{\circ} 28^{\prime} 9.81^{\prime \prime} \mathrm{E}$, altitude $3682 \mathrm{~m}$ ) receives photon 3 with a $400 \mathrm{~mm}$ diameter off-axis reflecting telescope. An integrated measurement system, consisting of an HWP, a QWP and a PBS, is assembled at the telescope's exit to measure any arbitrary state. Then, the photons are coupled in multi-mode fibers by a non-spherical lens. By selecting the appropriate fiber core and focal length, we compress the receiver field of view to $70 \mu \mathrm{rad}$, which directly improves the system's signal-to-noise ratio. In front of the non-spherical lens, two band-pass filters $\left(\Delta_{\mathrm{FWHM}}=80 \mathrm{~nm}\right)$ and one narrow-band interference filter $\left(\Delta_{\mathrm{FWHM}}=10 \mathrm{~nm}\right)$ are used to reduce background noises (IF shown in Fig. 22). Finally the photons are detected by the single-photon counting modules (SPCM) with ultra-low dark counts $\left(<20 \mathrm{~s}^{-1}\right)$. The noise that we observed, including the dark counts and ambient counts, is in total about $160 \mathrm{~s}^{-1}$ to $300 \mathrm{~s}^{-1}$. The noise mainly depends on the position of the moon and averagely we obtain about $200 \mathrm{~s}^{-1}$.

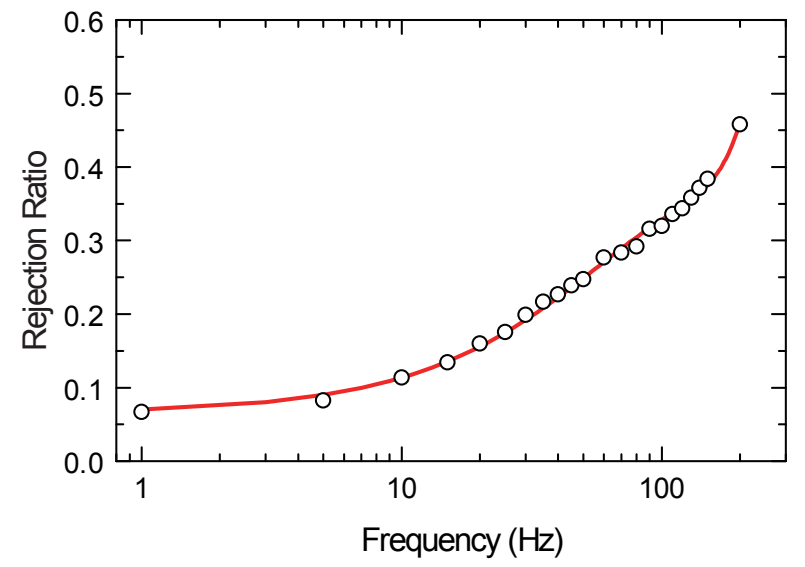

FIG. 3: The rejection ratio values as a function of the perturbation frequency. We perform this test indoors. An additional PI-FSM driven by a frequency-adjustable sinusoidal signal is used to induce the perturbation into fine tracking system of the transmitter. Here we define the ratio of the amplitudes between fine tracking on and off as rejection ratio. The solid line is a polynomial fit as the guide for eyes. The close-loop bandwidth of the fine tracking is defined as associated perturbation frequency at a rejection ratio of $0.5(-3 \mathrm{~dB})$. It is clear that the bandwidth is more than $150 \mathrm{~Hz}$.

\section{Acquiring, pointing and tracking system}

In addition to the optical design previously mentioned, we also equip an APT system to account for effects due to ground settlement, mechanical deformation, atmospheric turbulence, etc. As shown in Fig. 2a, on the sender Charlie's side, coaxial with the entangled photon 3 , there is a continuous green laser (532 nm, $200 \mathrm{mw}, 1.5 \mathrm{mrad}$ ) for system tracking and a pulsed infrared laser (1064 nm, $10 \mathrm{kHz}, 50 \mathrm{mw}, 200 \mu \mathrm{rad}$ ) for synchronization. On the receiver Bob's side, coaxial with the receiving telescope, there is a high-power beacon laser $(671 \mathrm{~nm}, 2 \mathrm{w}$, $200 \mu \mathrm{rad}$ ) for system tracking (Fig. 2. $)$. When the optical link was established for the first time, Charlie achieved acquiring by Global Positioning System (GPS) coordinates and light guide. At the same time, he fired the beacon light $(532 \mathrm{~nm}$ ) pointing to the receiver Bob. Bob then achieved acquisition and fired another beacon light (671 nm) pointing back to Charlie.

The tracking system is composed by a cascade closeloop control system (the blue and green arrows in Fig. 2 a and 2. . On Charlie's side, the beacon laser from the receiver Bob is detected by a wide-angle camera. With a feedback loop, the coarse alignment of the entire optical system is achieved by the two-dimensional rotatable platform in both azimuth and elevation (blue arrows in Fig. 2a). Similarly, the fine tracking indicated by the green arrows is achieved by the four-quadrant detector (QD) and fast steering mirror driven by piezo ceramics. Furthermore, the fine tracking system shares the same optical path as the quantum channel and is 
TABLE I: Fidelity of quantum teleportation over $97 \mathrm{~km}$. The data collection was accumulated for $14400 \mathrm{~s}$. The errors denote the statistical error, which is \pm 1 standard deviation.

\begin{tabular}{c|c}
\hline State & Fidelity \\
\hline \hline$H$ & $0.814 \pm 0.031$ \\
$V$ & $0.886 \pm 0.024$ \\
+ & $0.773 \pm 0.031$ \\
- & $0.781 \pm 0.031$ \\
$R$ & $0.808 \pm 0.026$ \\
$L$ & $0.760 \pm 0.027$ \\
\hline
\end{tabular}

later separated by a dichroic mirror (DM). Thus, a much higher tracking accuracy can be obtained. The closedloop bandwidth of the fine tracking is more than $150 \mathrm{~Hz}$ (see the inset in Fig. 3), which is sufficient to overcome most of the atmospheric turbulence A1. Finally, with this system design the tracking accuracy is better than $3.5 \mu \mathrm{rad}$ over the $97 \mathrm{~km}$ free-space link.

As indicated by the blue and green arrows in Fig. 2;, there are also coarse and fine tracking on the receiver Bob's side, by closed-loop control via the telescope's own rack and piezo ceramics. Since the main purpose of the tracking system at the receiver is to reduce the low frequency shaking due to ground settlement and passing vehicles, the closed loop bandwidth is about $10 \mathrm{~Hz}$. The APT system is designed for tracking an arbitrarily moving object, which can be directly utilized for a satellitebased QC experiment. In experiments between fixed locations, the first two steps, acquiring and pointing, do not need to be done every day.

In addition, we utilize a wireless Bridge for data transmission and classical communication between Alice and Bob. A high-accuracy Time-to-Digital Converter is used to independently record the arrival time of signals at both Alice's and Bob's station. A pulse per second produced by the GPS are added to synchronize the starting time. With the help of the pulsed synchronization laser, we achieve a time synchronization accuracy of better than $1 \mathrm{~ns}$ (see Supplementary Information for details).

\section{Results for quantum teleportation}

After debugging the entire system, we measured the channel loss in the Qinghai Lake district over $97 \mathrm{~km}$ horizontal atmospheric transmission at near ground levels. The measured link efficiency was between 35 and $53 \mathrm{~dB}$, in which $8 \mathrm{~dB}$ was due to the imperfect optics and finite collection efficiency and 8 to $12 \mathrm{~dB}$ was due to atmospheric loss. The geometric attenuation due to the beam spreading wider than the aperture of the receiver telescope was between 19 to $33 \mathrm{~dB}$, corresponding to a far-field spot size of between 3.5 and $17.9 \mathrm{~m}$, depending on weather conditions. With a tracking accuracy of $3.5 \mu \mathrm{rad}(0.34 \mathrm{~m}$ at the receiver), we had stable count rates for single photons. We obtained 1171 coincidences during an effective time of $14400 \mathrm{~s}$. The average channel attenuation was about $44 \mathrm{~dB}$, and the time accuracy was better than 1 ns. We selected linear polarization states $|H\rangle,|V\rangle$ and $| \pm\rangle=(|H\rangle \pm|V\rangle) / \sqrt{2}$, circular polarization states $|R\rangle=(|H\rangle+i|V\rangle) / \sqrt{2}$ and $|L\rangle=(|H\rangle-i|V\rangle) / \sqrt{2}$ as the initial states to be teleported. The final data results are shown in Table [] The experimental results for teleportation fidelity for different initial states range from $76 \%$ to $89 \%$, with an overall average fidelity of $80 \%$. The fidelities for the six teleported states were all well beyond the classical limit of $2 / 3$.

\section{Two-link entanglement distribution}

In the teleportation experiment, Alice and Charlie are close to each other. A more common situation would be that Alice is also far away from Charlie. In this case, distribution of entanglement between Alice and Bob is a prerequisite towards quantum teleportation. A feasible solution is to distribute the entanglement to Alice and Bob by Charlie via a two-link channel. To demonstrate the two-link entanglement distribution, we move the entanglement source close to the middle of the free-space channel, an island in the middle of the Qinghai Lake $\left(36^{\circ} 51^{\prime} 38.75^{\prime \prime} \mathrm{N}, 100^{\circ} 8^{\prime} 15.22^{\prime \prime} \mathrm{E}\right)$, as shown in Fig. 4. To show the novelty of entanglement distribution, Bob move his receiving platform to a local Tibetan family (GongHe $36^{\circ} 32^{\prime} 20.66^{\prime \prime} \mathrm{N}, 100^{\circ} 33^{\prime} 45.38^{\prime \prime} \mathrm{E}$ ) next to the GuanJing, where it is not possible for Bob to see Alice directly. Charlie first prepares the entangled photon pairs in state $\left|\Phi^{+}\right\rangle$. Then the entangled photon pairs are sent to Alice and Bob via two telescopes each mounted on a two-dimensional rotatable platform. The distance between Charlie and two receivers is $51.2 \mathrm{~km}$ (Alice) and $52.2 \mathrm{~km}$ (Bob), and the distance between Alice and Bob is $101.8 \mathrm{~km}$.

The same APT system as in the teleportation experiment is used between Alice and Charlie, as well as between Bob and Charlie. Entangled photons are collected by telescopes on both sides. In contrast to the $400 \mathrm{~mm}$ off-axis reflecting telescope used on Bob's side, Alice uses a $600 \mathrm{~mm}$ Cassegrain telescope to collect the photons. In order to confirm the successful entanglement distribution between the two receivers, we measure the $S$ parameter in the Clauser-HorneShimony-Holt (CHSH) type Bell's inequality [27]: $S=$ $\left|E\left(\varphi_{A}, \varphi_{B}\right)-E\left(\varphi_{A}, \varphi_{B}^{\prime}\right)-E\left(\varphi_{A}^{\prime}, \varphi_{B}\right)-E\left(\varphi_{A}^{\prime}, \varphi_{B}^{\prime}\right)\right|$, where $E\left(\varphi_{A}, \varphi_{B}\right)$ is the correlation function, and $\varphi_{A}$ and $\varphi_{A}^{\prime}\left(\varphi_{B}\right.$ and $\left.\varphi_{B}^{\prime}\right)$ are the measured polarization bases of the photon in Alice's (Bob's) hand. In the measurement, the polarization settings are $(0, \pi / 8),(0,3 \pi / 8),(\pi / 4$, $\pi / 8)$ and $(\pi / 4,3 \pi / 8)$ and randomly selected. Each measuring module consists of a HWP, a fast electro optical modulator (EOM) and its modulation logical circuit, a PBS, and two fiber-coupled SPCMs. The optical axes of the EOM are set at $22.5^{\circ}$ to act as HWPs which transform the diagonal (antidiagonal) polarization 


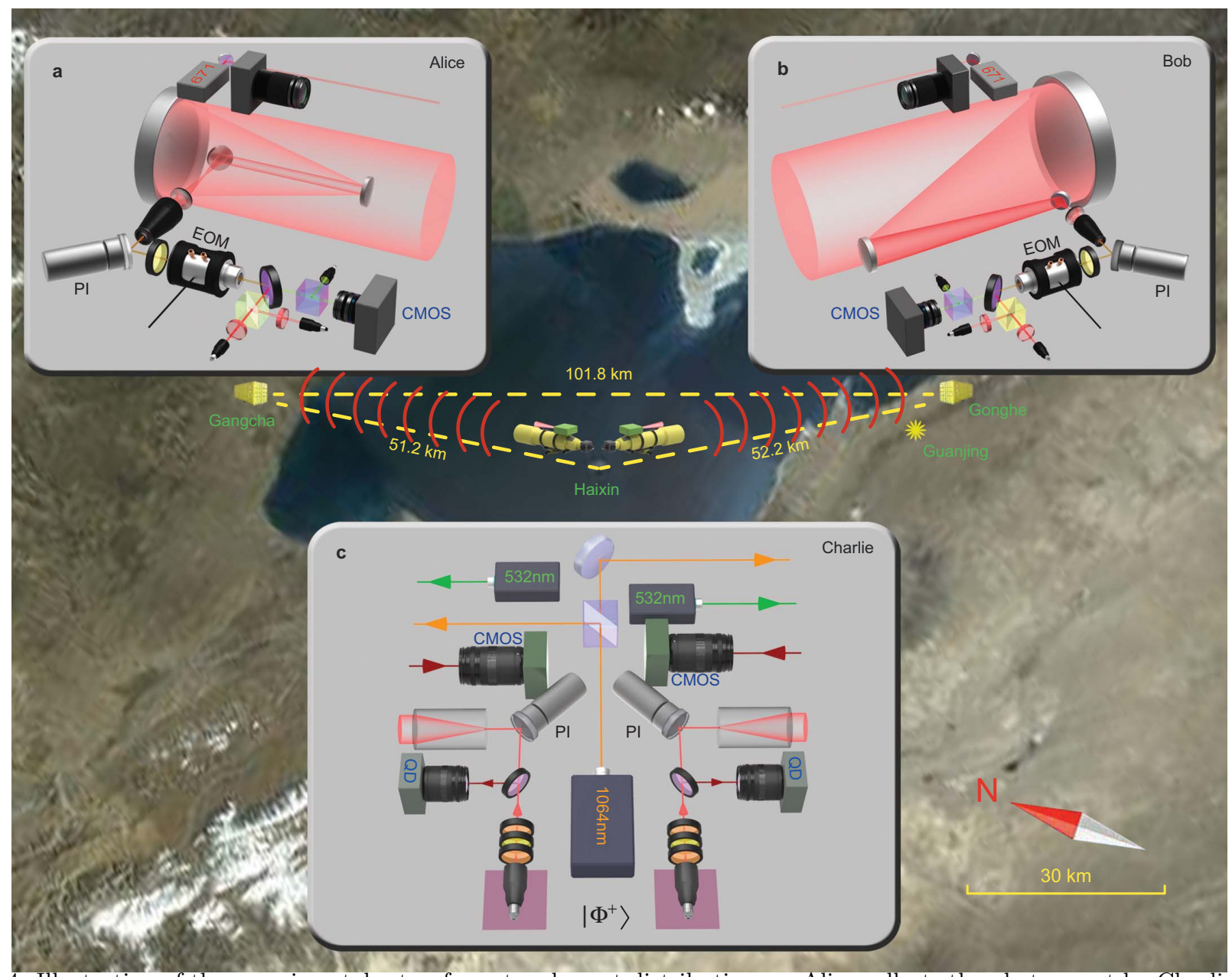

FIG. 4: Illustration of the experimental setup for entanglement distribution. a, Alice collects the photon sent by Charlie using a $600 \mathrm{~mm}$ Cassegrain telescope. b At the receiver Bob, the photon beams are collected by a $400 \mathrm{~mm}$ off-axis reflecting telescope. c An entangled photon pair $\left(\left|\Phi^{+}\right\rangle\right)$is created by Charlie at the centre island of Qinghai Lake (Haixin). Each photons is then guided to a telescope mounted on a two-dimensional rotatable platform. Then Charlie distributes the two entangled photon to Alice and Bob. The same APT system as in the teleportation experiment is used to create the two-link quantum channel between Alice and Charlie and between Bob and Charlie. After receiving the photons, Alice and Bob guide the photons to the detection module by an optical system. This module consists of an EOM, a PBS and two fiber coupled SPCMs.

into a horizontal (vertical) polarization and viceversa when half-wave voltages are applied. The EOMs act as absent wave plates when zero-wave voltages are imposed. Quantum random number generators (QRNG) are used to produce the random digital series between zero-wave and half-wave voltage. Together with the HWP, which is set at $0^{\circ}$ at Alice's side and $11.25^{\circ}$ at Bob's side, and the QRNG, the EOM randomly switches between the two desired measurement bases - 0 and $\pi / 4$ for Alice and $\pi / 8$ and $3 \pi / 8$ for Bob.

Finally, we obtained 208 coincidences during an effective time of $32000 \mathrm{~s}$. Comparing with the counts of our entanglement source, the channel attenuation varied from $66 \mathrm{~dB}$ to $85 \mathrm{~dB}$ with an average value of $79.5 \mathrm{~dB}$. For $20 \mathrm{~cm}$ aperture satellite optics at an orbit height of $600 \mathrm{~km}$ and $1 \mathrm{~m}$ aperture receiving optics, the total loss for a two-downlink channel between a satellite and two grounds stations is typically about $75 \mathrm{~dB}$. The mea- sured correlation functions (shown in Fig. 5 ) resulted in $S=2.51 \pm 0.21$, which violates Bell's inequality by 2.4 standard deviations.

In addition, our experiment closed the locality loophole. The entangled photon pairs were distributed along two opposite directions to Alice and Bob. The distance between Alice and Bob is $101.8 \mathrm{~km}$, which takes $340 \mu \mathrm{s}$ for light to traverse, and the path difference between Charlie and Alice and between Charlie and Bob is $1 \mathrm{~km}$, which results in a $3 \mu$ s delay between the two measurement events. Thus, Alice and Bob are space-like separated. Furthermore, the two receivers used fast EOMs to switch between the two possible polarization bases. The two EOMs were controlled by two independent QRNGs, each of which generates a random number every $20 \mu \mathrm{s}$ (less than $340 \mu \mathrm{s}$ ). Thus the measurement setting choices are also space-like separated. Hence, the locality loophole is closed. 


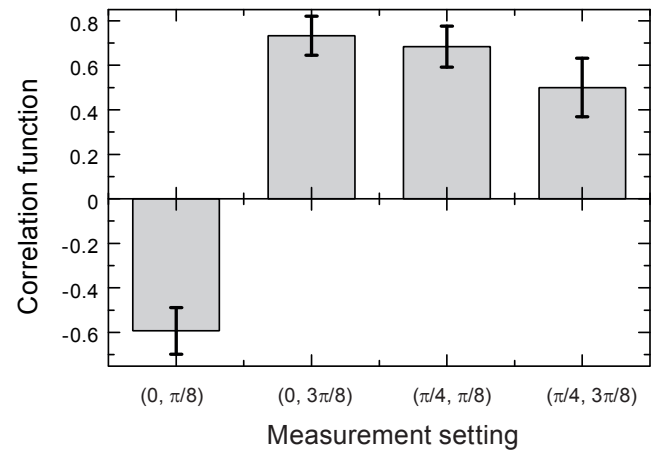

FIG. 5: Correlation functions of a CHSH-type Bell's inequality for entanglement distribution. The measurement setting $\left(\varphi_{A}, \varphi_{B}\right)$ represents the measured polarization bases of photons by Alice and Bob, respectively. Error bars represent statistical errors, which are \pm 1 s.d.

\section{Discussion}

In this work, based on multi-photon entanglement, we experimentally realized free-space quantum teleportation for an independent quibit over a $35-53 \mathrm{~dB}$ loss onelink channel. In comparison with previous multi-photon experiments 19, 20, we have enhanced the transmission distance by two orders of magnitude to $97 \mathrm{~km}$. Furthermore, we demonstrated the successful distribution of an entangled photon pair over a two-link free-space optical channel to two receivers separated by more than $100 \mathrm{~km}$. In contrast to previous long-distance free-space experiments with an entangled photon pair using only one-link channels [23, 24], our two-link experiment requires tracking and synchronization with three different locations. Our two-link experiment, which most comparable with satellite-to-ground quantum entanglement distribution, has achieved a distance between two receivers by an order of magnitude larger than in all previous experiments. This shows the feasibility of achieving two-link quantum teleportation with either the original scheme 2, 11] or the modified scheme 25, 26. Our results show that even with a high-loss ground-to-satellite uplink channel, or satellite-to-ground two-downlink channel, quantum teleportation and entanglement distribution can be realized. Furthermore, our APT system can be used to track an arbitrarily moving object with high frequency and high accuracy, which is essential for future satellite-based ultralong-distance QC. We hope our experiment will boost the tests of the quantum fundations on a global scale.

This work has been supported by the NNSF of China, the CAS, the National Fundamental Research Program (under Grant No. 2011CB921300).
[1] J.-W. Pan et al., Multi-photon entanglement and interferometry. Rev. Mod. Phys., in press. Preprint available at http://arxiv.org/abs/0805.2853v2.

[2] C. H. Bennett et al., Phys. Rev. Lett. 70, 1895 (1993).

[3] H. J. Briegel, W. Dur, J. I. Cirac and P. Zoller, Phys. Rev. Lett. 81, 5932 (1998).

[4] A. K. Ekert, Phys. Rev. Lett. 67, 661 (1991).

[5] J. S. Bell, Physics (Long Island City, N.Y.) 1, 195 (1964).

[6] S. J. Freedman and J. F. Clauser, Phys. Rev. Lett. 28, 938 (1972).

[7] A. Aspect, P. Grangier and G. Roger, Phys. Rev. Lett. 47, 460 (1981).

[8] G. Weihs et al., Phys. Rev. Lett. 81, 5039 (1998).

[9] R. J. Hughes, J. E. Nordholt, D. Derkacs and C. G. Peterson, New J. Phys. 4, 43 (2002).

[10] C. Kurtsiefer et al., Nature 419, 450 (2002).

[11] D. Bouwmeester et al., Nature 390, 575 (1997).

[12] J.-W. Pan, D. Bouwmeester, H. Weinfurter and A. Zeilinger, Phys. Rev. Lett. 80, 3891 (1998).

[13] Z. Zhao et al., Nature 430, 54 (2004).

[14] Q. Zhang et al., Nature Physics 2, 678 (2006).

[15] I. Marcikic et al., Phys. Rev. Lett. 93, 180502 (2004).

[16] H. Hübel et al., Optics Express 15, 7853 (2007).

[17] Q. Zhang et al., Optics Express 16, 5776 (2008).

[18] J. F. Dynes et al., Optics Express 17, 11440 (2009).

[19] I. Marcikic et al., Nature 421, 509 (2003).

[20] R. Ursin et al., Nature 430, 849 (2004).

[21] M. Aspelmeyer et al., Science 301, 621 (2003).

[22] C.-Z. Peng et al., Phys. Rev. lett. 94, 150501 (2005).

[23] R. Ursin et al., Nature Physics 3, 481 (2007).

[24] A. Fedrizzi et al., Nature Physics 5, 389 (2009).
[25] D. Boschi et al., Phys. Rev. Lett. 80, 1121 (1998).

[26] X.-M. Jin et al., Nature Photonics 4, 376 (2010).

[27] J. F. Clauser, M. A. Home, A. Shimony and R. A. Holt, Phys. Rev. Lett. 23, 880 (1969).

[28] C.-Y. Lu et al., Nature Physics 3, 91 (2007).

[29] S. Popescu, Phys. Rev. Lett. 72, 797 (1994).

[30] X.-C. Yao et al., Nature Photonics 6, 225 (2012).

[31] P. G. Kwiat et al., Phys. Rev. Lett. 75, 4337 (1995).

[32] Y.-H. Kim et al., Phys. Rev. A 67, 010301 (2003).

[33] J. W. Strohbehn, Laser beam propagation in the atmosphere. Berlin: Springer-Verlag (1978).

\section{Appendix}

\section{A.I. ACQUIRING, POINTING AND TRACKING SYSTEM}

Considering a total optical channel of almost $100 \mathrm{~km}$ at near-surface atmosphere, one of the most crucial points is that how to guarantee the stability of the efficiency of the quantum channel. Generally speaking, there are mainly two factors depraving the stability of the quantum channel. One is the instabilities of transmitting platforms mainly caused by ground settlement and mechanical stress relieving, which induce quantum-signal beam deviation slowly and drastically. The other one is angle-of-arrival (AOA) fluctuations of quantum-signal beam caused by atmospheric turbulence, which is at fre- 


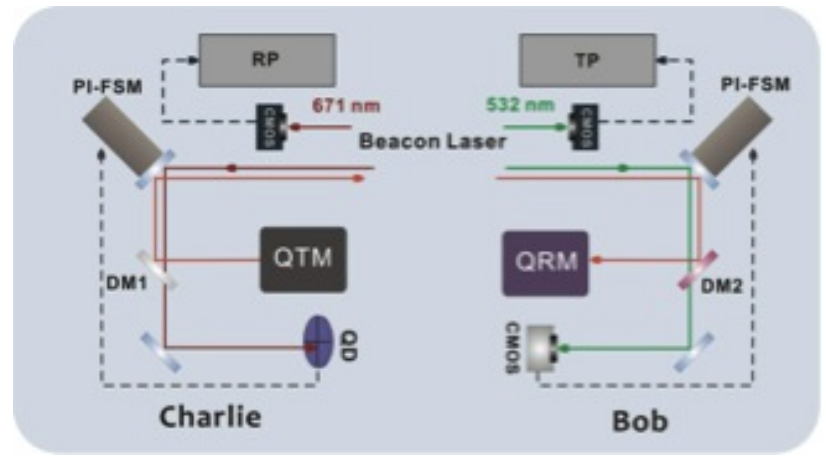

FIG. A1: A sketch of the tracking systems equipped on Charlie's and Bob's station. DM1: dichroic mirror ( $\mathrm{T}$ : $671 \mathrm{~nm}$; R: $788 \mathrm{~nm}$ ); DM2: dichroic mirror (T: $532 \mathrm{~nm}$; R 788 $\mathrm{nm})$; QD: four-quadrant detector; QTM: quantum transmitting module (see Fig. 2 in the main text for detail); QRM: quantum receiving module (see Fig. 2 in the main text for details); RP: the two-dimensional rotatable platform; TP: telescopes rack.

quency of less than $100 \mathrm{~Hz}$. For solving above problems, we equip an acquiring, pointing and tracking (APT) system on both transmitting and receiving sides.

\section{A. APT system for quantum teleportation}

On the sender Charlie's side, as shown in Fig. A1, the APT is composed of a two-stage system, namely coarse tracking and fine tracking respectively. For the coarse tracking system, the actuator is a two-dimensional rotatable platform in both direction in the azimuth and pitching (blue arrows in Fig. 2a in the main text). A wide-angle complementary metal oxide semiconductor (CMOS) camera is used to detect the beacon laser coming from the receiver Bob to provide a feedback loop for coarse tracking. Considering the main function of tracking system is to perform the coarse alignment with the receiver and offset the disturbance due to ground settlement and mechanical deformation, the coarse tracking is designed with $30 \mathrm{mrad}$ of tracking field-of-view (FOV), $30 \mu \mathrm{rad}$ of tracking accuracy and $10 \mathrm{~Hz}$ of closed-loop bandwidth. Furthermore, for fine pointing to the receiver and restraining the beam quiver due to atmospheric turbulence, we implement the fine tracking exploiting a fourquadrant detector (QD) for providing a feedback loop, a fast steering mirror (FSM) driven by piezo ceramics for an actuator. Different with the coarse tracking, the fine tracking system shares the same optical path as the quantum channel and is later separated by a dichroic mirror (DM) (see Fig. A1 and Fig. 2a in the main text). Thus, a much higher tracking accuracy $(3 \mu \mathrm{rad})$ can be obtained. The response frequency of the QDs is $100 \mathrm{kHz}$. With the optimal control software with proportion-integrationdifferentiation (PID) arithmetic, the closed-loop bandwidth of the fine tracking is more than $150 \mathrm{~Hz}$ (see Fig. 3

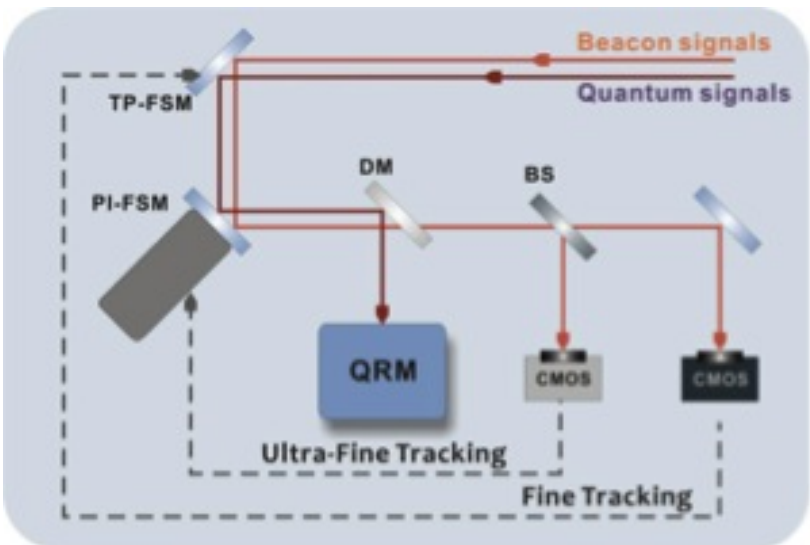

FIG. A2: A sketch of fine tracking system equipped on Alice's telescope in QED experiment. DM: dichroic mirror; BS: beam splitter; QAM: quantum receiver module (see the details in Fig. 4a in the main text).

in the main text), which is sufficient to overcome most of the atmospheric turbulence [A1]. Finally, with above designed system we get the tracking accuracy better than $3.5 \mu$ rad over $97 \mathrm{~km}$ free-space link.

At the receiver Bob's side, there are also coarse and fine tracking systems, by close-loop control via the telescope's own rack and piezo ceramics. Since the main purpose of the tracking system at the receiver is to reduce the low frequency shaking due to ground settlement and passing vehicles, the closed-loop bandwidth is about $10 \mathrm{~Hz}$.

The brief introduction on the procedure of establishing the free-space quantum optical link is as follows: First, Charlie achieved acquiring by Global Positioning System (GPS) coordinates and light guide of Bob; Then, a continuous green laser $(532 \mathrm{~nm}, 200 \mathrm{~mW}, 1.5 \mathrm{mrad})$ coaxial with the entangled photon 3, is sent from Charlie to Bob; Third, when Bob acquires and tracks the green laser, he fires a high-power beacon laser $(671 \mathrm{~nm}, 2 \mathrm{~W}, 200 \mu \mathrm{rad})$ to Charlie for her tracking system; Fourth, utilizing the beacon laser coming from Bob, Charlie turns on her APT system; Finally, after activating the APT systems of both sides, Charlie transmits the entangled photon 3 and a pulsed synchronization laser $(1064 \mathrm{~nm}, 10 \mathrm{kHz}, 50 \mathrm{~mW}$, $200 \mu \mathrm{rad})$ to Bob. The APT system is designed for tracking an arbitrarily moving object, which can be directly utilized for a satellite-based quantum communication experiment. In experiments between fixed locations, like the experiment presented here, the first two steps, acquiring and pointing, do not need to be done every day.

\section{B. APT system for entanglement distribution}

For the experiment of long-distance quantum entanglement distribution (QED) over two-link free-space channel, the APT systems on Charlie's (the transmitter) and Bob's (the receiver at Gonghe) station are almost iden- 
tical with the ones used in the teleportation experiment. To obtain a more efficient and stable free-space optical channel, we employ a larger optical telescope with 600 mm diameter and a high-speed APT system at Alice's (the other receiver) station.

In order to solve the problems of dithering of beam light caused by the low order effects of atmospheric turbulence, we have made great efforts on improving the existing APT of Alice's receiver telescope. The main improvement is that we divide the original fine tracking system into two levels, called fine tracking and ultra-fine tracking (see Fig. A2). For the fine tracking, a CMOS camera with $400 \mathrm{~Hz}$ of sampling rate and $15 \mathrm{um}$ of per pixel size is used. An indigenous FSM with a shrapnel structure, namely TP-FSM is used as an actuator in the fine tracking system. The overall closed-loop bandwidth is about $15 \mathrm{~Hz}$. Considering the equivalent focal length is $4.3 \mathrm{~m}$, the fine tracking system provide a relatively large FOV. For the ultra-fine tracking, a higher sampling rate $(2 \mathrm{kHz})$ CMOS camera is exploied. And due to the longer equivalent focal lengths $(7 \mathrm{~m})$ and a higher performance actuator (FSM based on piezo, namely PI-FSM), the ultra-fine tracking provide a higher tracking accuracy $(2 \mu \mathrm{rad})$ and closed-loop band-with $(75 \mathrm{~Hz})$. But since the very narrow FOV of the ultra-fine tracking, we should run the tracking system in combination mode in which the two level tracking system are both turned on to obtain the best tracking effect.

Beside the tip tilt error due to the turbulence, there is also higher order effect by the turbulence, which expressed as beam spread. As the telescope aperture we used at the receiver is much smaller than the far-field spot size, any speckle effects results more in geometric attenuation than efficiency fluctuation, which cannot be directly observed with our system. To narrow the far field spot, one needs to increase the tracking bandwidth to more than $\mathrm{kHz}$ with new technologies, for example an adaptive optics (AO) system.

\section{A.II. SYNCHRONIZATION ACCURACY}

In the experiment, as shown in Fig. 2 and Fig. 4 in the main text, a pulsed infrared laser $(1064 \mathrm{~nm}, 10 \mathrm{kHz}$,
$50 \mathrm{mw}, 200 \mu \mathrm{rad})$ is used for synchronization. The pulse length of the synchronization laser is $2.65 \mathrm{~ns}$ (FWHM), with the rising edge of 2 ns. A wireless Bridge is used for data transmission and classical communication between Alice and Bob. A pulse per second by the GPS are added to synchronize the starting time.

First we collect the laser with multi-mode fibers (200 $\mu \mathrm{m}$, NA 0.22$)$ and then guide the light into fast photoreceivers at both receiving sides. Then we deal with the forefront of detected signals with constant fraction discriminator (CFD) technique and utilize a high-accuracy Time-to-Digital Converter (TDC) with 100 ps time resolution precision to record the arrival time of the synchronization signals at both Alice's and Bob's station.

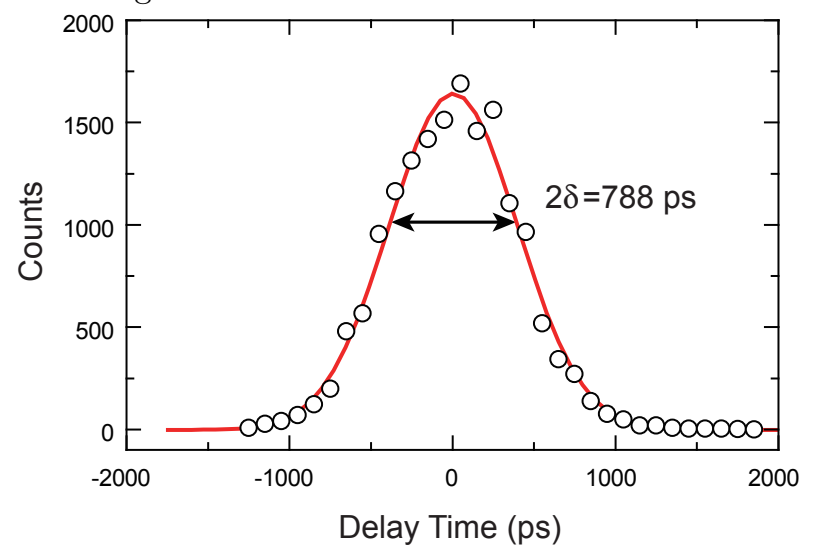

FIG. A3: A typical recorded arrival time of the $1064 \mathrm{~nm}$ laser signals at both stations. Synchronization accuracy of $2 \delta=788(13)$ ps was observed, $\delta$ refers to the width of the gaussian fit (solid line).

Through the above methods, we had overcome most of the time jitter caused by the laser pulse itself and energy shaking. In the experiment, the quantum signal was collected by SPCM and an additional time jitter of $350 \mathrm{~ns}$ has to be considered. Finally we achieved time synchronization for the quantum channel better than $1 \mathrm{~ns}$ over $100 \mathrm{~km}$ distance (See Fig. A3) 